\title{
EXCITON ABSORPTION IN GaN
}

\author{
M. SOBOL AND W. BardyszewsKi
}

Institute of Theoretical Physics, Warsaw University, Hoża 69, 00-681 Warsaw, Poland

The absorption coefficient of the wurtzite-type $\mathrm{GaN}$ is described in terms of three almost totally decoupled excitons $A, B, C$ associated with split valence bands of $\Gamma_{9}, \Gamma_{7}$ and $\Gamma_{7}$ symmetries using a spherically symmetric approximation for the standard effective-mass Hamiltonian. The excitonic equations are solved within the subspace of optically active states of $s$-symmetry. The effects of crystal field and spin-orbit coupling are discussed. The results show that the spherical approximation is in perfect agreement with experimental findings.

PACS numbers: $71.35 .-\mathrm{y}, 78.20 . \mathrm{Bh}, 78.66 . \mathrm{Fd}$

\section{Introduction}

The group of III-V nitrides, such as GaN, usually crystallize in the wurtzite-type structure. These materials have recently attracted much attention, since they have an enormous potential for various applications such as blue light-emitting diodes and ultraviolet photodetectors. Gallium nitride belongs to the group of direct band gap semiconductors. In general, description of optical phenomena requires the detailed knowledge of electron and hole spectra near the center of the Brillouin zone. Thus the standard $k \cdot p$ valence band Hamiltonian, which is obtained by using irreducible representations of the wurtzite space group $C_{6 v}^{4}$ at the $\Gamma$ point, provides an appropriate basis of the analysis of electronic properties of the material. The purpose of this study is to provide a theoretical understanding of absorption processes related to the three almost decoupled $A, B$, and $C$ excitons corresponding to the split valence band subbands $\Gamma_{9}, \Gamma_{7}$ and $\Gamma_{7}$ at the $\Gamma$ point. It is commonly accepted that these excitons have almost identical binding energies although there is a considerable discrepancy in the published experimental data regarding their actual values. To understand these data a simplified multi-band exciton model has been developed using the approach proposed by Baldereschi and Lipari [1] for zinc-blende structure semiconductors. We show that the zeroth order approximation retaining merely the spherically symmetric part of the electron-hole kinetic energy describes well the recently measured absorption spectrum in GaN. 


\section{Exciton Hamiltonian}

The interband absorption in GaN is determined by the electron-hole pair $s$-type wave function in the vicinity of the band extrema. The Hamiltonian describing the relative electron-hole motion is given by

$$
\mathcal{H}=\mathcal{H}_{0}-\frac{e^{2}}{\epsilon_{0} r}
$$

where an isotropic Coulomb interaction screening with the dielectric constant $\epsilon_{0}$ is assumed. The free electron-hole pair Hamiltonian is given by

$$
\mathcal{H}_{0}=\frac{p^{2}}{2 m_{\mathrm{e}}^{*}} \widehat{I}-\widehat{H}(\boldsymbol{p})
$$

where the first term describes the electron motion and is diagonal in the valence subbands indices. The second term takes into account the three subbands in the vicinity of the valence band edge and is given by the Rashba-Sheka-Pikus (RSP) [2] matrix Hamiltonian

$$
\begin{aligned}
\widehat{H}(p) & =\Delta_{1} \widehat{J}_{z}^{2}+\Delta_{2} \widehat{J}_{z} \sigma_{z}+\sqrt{2} \Delta_{3}\left(\widehat{J}_{+} \sigma_{-}+\widehat{J}_{-} \sigma_{+}\right)+\left(A_{1}+A_{3} \widehat{J}_{z}^{2}\right) p_{z}^{2} \\
& +\left(A_{2}+A_{4} \widehat{J}_{z}^{2}\right)\left(p_{x}^{2}+p_{y}^{2}\right)-A_{5}\left(\widehat{J}_{+}^{2} p_{-}^{2}+\widehat{J}_{-}^{2} p_{+}^{2}\right) \\
& -2 A_{6} p_{z}\left(\left[\widehat{J}_{z}, \widehat{J}_{+}\right]_{+} p_{-}+\left[\widehat{J}_{z}, \widehat{J}_{-}\right]_{+} p_{+}\right),
\end{aligned}
$$

where the terms linear in $p$ have been neglected. The matrices $\widehat{J}_{x, y, z}$ represent $J=1$ components of the angular momentum operator. Furthermore $\widehat{J}_{ \pm}=$ $(1 / \sqrt{2})\left(\widehat{J}_{x} \pm \mathrm{i} \widehat{J}_{y}\right), \quad p_{ \pm}=p_{x} \pm \mathrm{i} p_{y}$ and $\sigma_{ \pm}=(1 / 2)\left(\sigma_{x} \pm \mathrm{i} \sigma_{y}\right)$, where $\sigma_{x, y, z}$ are standard Pauli matrices. The brackets $\left[\widehat{J}_{z}, \widehat{J}_{-}\right]_{+}$denote a symmetrized product. The constants $A_{1 \ldots .6}$ and $\Delta_{1,2,3}$ are extracted either from experimental data or from $a b$ initio band structure calculations. Despite existing differences regarding the values of those parameters, all published sources seem to agree that the regular crystal approximation requiring $\Delta_{2}=\Delta_{3}, A_{3}=A_{2}-A_{1}=-2 A_{4}$ and $A_{6}=\left(A_{3}+4 A_{5}\right) / \sqrt{2}$, is well justified for GaN. The additional constraint $A_{5}=\left(A_{1}-A_{2}\right) / 2$ makes the $p$-dependent part of RSP Hamiltonian ideally spherically symmetric. According to the most frequently cited values of band structure parameters, this last identity holds almost exactly in GaN. Consequently the free electron-hole Hamiltonian can be approximately written as

$$
\begin{gathered}
\mathcal{H}_{0}(p)=-\Delta_{1} J_{z}^{2}-\Delta_{2} \boldsymbol{J} \cdot \boldsymbol{\sigma}+\left[\frac{1}{2 m_{\mathrm{e}}^{*}}-\frac{1}{3}\left(A_{1}+2 A_{2}\right)\right] \widehat{I} p^{2} \\
+\left(A_{1}-A_{2}\right)\left[(\boldsymbol{p} \cdot \boldsymbol{J})^{2}-\frac{2}{3} \widehat{I} p^{2}\right] .
\end{gathered}
$$

Here the first and second terms describe the crystal field splitting and spin-orbit coupling respectively while the next two represent $s$-symmetric and $d$-symmetric parts of the kinetic energy and will be denoted by $\widehat{H}^{s}$ and $\widehat{H}^{d}$ correspondingly.

In the absence of the crystal field splitting the above Hamiltonian can be easily block-diagonalized in the representation of full angular momentum constructed by first adding the spin $s=\sigma / 2$ to the atomic angular momentum $\boldsymbol{J}: \boldsymbol{I}=\boldsymbol{s}+\boldsymbol{J}$, and then adding the exciton envelope function angular momentum $\boldsymbol{L}$. Denoting 
the resulting states by $\left|L, I ; F, F_{z}\right\rangle$, we remark that while $F$ and $F_{z}$ are in this case good quantum numbers, the $\widehat{H}^{d}$ part of the Hamiltonian mixes states with $\Delta L= \pm 2$ and $\Delta I= \pm 1$. Unfortunately, unlike in zinc-blende compounds, the $d$-term is not much smaller than the symmetric $s$-term and cannot be justifiably neglected at this stage. The situation changes when the crystal field splitting is included. The crystal field breaks the spherical symmetry and mixes states with different $F$ and $I$ but leaves the envelope angular momentum $L$ unchanged. It may be then justified to first solve the problem without the $\widehat{H}^{d}$ and then include it as a perturbation. In this case the first correction to the lowest excitonic levels comes from the second order of the perturbation theory for nondegenerate states. The correction to the higher lying levels may be more important. In particular the discrete states degenerate with the continuum belonging of other excitons may be additionally broadened [1]. In the present paper we neglect the effects of the $d$-term altogether.

Specializing to the optically active $L=0$ states we observe that due to the total isotropy of $\widehat{H}^{s}$, we can easily diagonalize the excitonic Hamiltonian when $\widehat{H}^{d}$ is neglected. As a result we obtain three spectral series for exciton $A$ associated with energy level equal to the energy gap $E_{A}=E_{\mathrm{g}}$, and for excitons $B$ and $C$ associated with energy levels $E_{B, C}=E_{\mathrm{g}}+\left[\Delta_{1}+3 \Delta_{2} \mp \sqrt{\left(\Delta_{1}-\Delta_{2}\right)^{2}+8 \Delta_{2}^{2}}\right] / 2$ respectively. The eigenstates for exciton $A$ are given simply by $\left|\Psi_{A}\right\rangle=$ $f(r)\left|L=0, I=3 / 2 ; F=3 / 2, F_{z}=3 / 2\right\rangle$ while the other two are linear combinations $\left|\Psi_{B, C}\right\rangle=f(r)\left(\alpha_{B, C}\left|L=0, I=1 / 2 ; F=1 / 2, F_{z}=1 / 2\right\rangle+\beta_{B, C} \mid L=0\right.$, $\left.\left.I=3 / 2 ; F=3 / 2, F_{z}=1 / 2\right\rangle\right)$. The envelope function $f(r)$ in each case is found from the Elliot theory [3] with the effective reduced mass

$$
\frac{1}{\mu}=\frac{1}{m_{\mathrm{e}}^{*}}-\frac{2 m_{0}}{\hbar^{2}}\left[\frac{1}{3}\left(A_{1}+2 A_{2}\right)\right]
$$

resulting in equal binding energies for all three excitons.

\section{Results and discussion}

While there is a reasonable consensus for the values of $A_{i}$ band parameters in gallium nitride, the magnitude of the crystal field and spin-orbit splitting is still under debate. The experimental results for epitaxial layers are very often biased by an unknown built-in strain from the lattice mismatch with the substrate. On the theoretical side it has been long known that the band structure calculations using the local density theory do not provide accurate enough energy gaps. Recently the results of optical measurements of high quality, homoepitaxial, i.e. unstrained layers of GaN have been published [4]. In order to test our simplified spherical model we have calculated the near edge absorption spectrum using parameters of Ref. [5]. The comparison between the theoretical model and the experimental data taken from Ref. [4] is given in Fig. 1. It has to be stressed that the only adjusted parameters in our calculation were the position of the first exciton maximum and the broadening constants. We have assumed Lorentzian line-shapes with the HWHM equal to $1 \mathrm{meV}$ for all discrete lines as well as for the continuum edge. The positions of excitons $A$ and $B$ and their relative intensities result directly from our model and seem to be in remarkably good agreement with the experiment. 


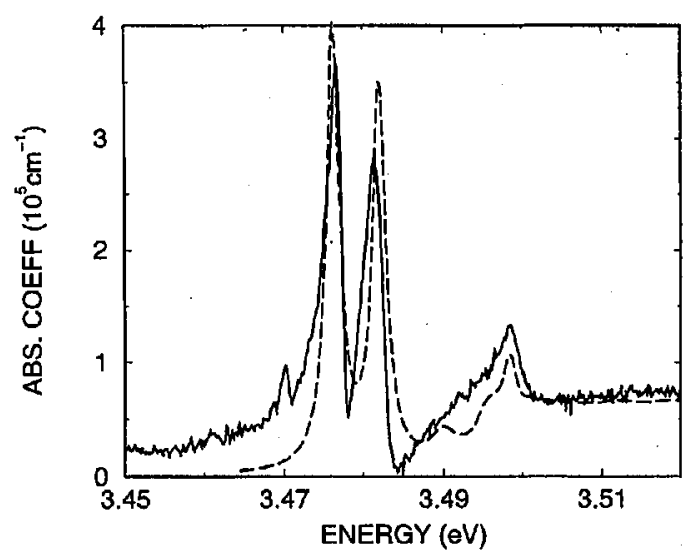

Fig. 1. Experimental (solid line) and theoretical (dashed line) near-edge absorption spectrum. Experimental data are taken from Ref. [4].

A short discussion is in order concerning the effective reduced mass and the binding energies. There is a great number of experimental values of binding energies obtained with different spectroscopic techniques. The most reliable estimates seem to be those inferring the binding energy from the relative position of the $n=1$ and $n=2$ excitonic levels. The reported values for $A$ excitons can be divided into two groups. Values close to $20 \mathrm{meV}$ as for example in Ref. [6] $\left(E_{\mathrm{b}}=21 \mathrm{meV}\right.$ from reflectance at $T=10 \mathrm{~K})$, Ref. [7] $\left(E_{\mathrm{b}}=20 \mathrm{meV}\right.$ from photoluminescence at $T=2 \mathrm{~K})$, Ref. [8] $\left(E_{\mathrm{b}}=20.4 \mathrm{meV}\right.$ from transmission at $\left.T=295 \mathrm{~K}\right)$ or in Ref. [9] $\left(E_{\mathrm{b}}=18.3 \mathrm{meV}\right.$ from photoluminescence at $\left.T=10-150 \mathrm{~K}\right)$ are in striking contradiction to much higher values reported in Ref. [10] $\left(E_{\mathrm{b}}=27.1 \mathrm{meV}\right.$ from two-photon spectroscopy at $T=7 \mathrm{~K})$ or Ref. [11] $\left(E_{\mathrm{b}}=26.7 \mathrm{meV}\right.$ from calorimetric spectroscopy at $T=45 \mathrm{mK}$ ).

The latter value is very often advocated in connection with the large effective mass of holes in the high-symmetry directions. The spherical model suggests on the other hand that the spherically symmetrized effective mass deduced from Eq. (5) should be used which for the present values of band parameters yields $m_{\mathrm{h}}=0.36$. Assuming $\epsilon_{0}=9.6$ and $m_{e}^{*}=0.19 m_{0}$ we obtain $E_{\mathrm{b}}=18.3 \mathrm{meV}$ in agreement with the lower values reported in the literature. Although the spherical model points to the smaller exciton binding energies we feel that this question requires further investigation.

In conclusion we have presented a simplified spherical model of excitons in GaN giving equal binding energies for all three excitons. A simple expression for the spherically averaged effective mass has been derived which gives binding energies in agreement with some experimental data. Using our model we could successfully describe the absorption spectra in unstrained homoepitaxial layers of gallium nitride. 


\section{References}

[1] A. Baldèreschi, N.C. Lipari, Phys. Rev. B 3, 439 (1971).

[2] G.L. Bir, G.E. Pikus, Symmetry and Strain-Induced Effects in Semiconductors, Wiley, New York 1974.

[3] R.J. Elliot, Phys. Rev. 108, 1384 (1957).

[4] R. Stępniewski, K.P. Korona, A. Wysmołek, J.M. Baranowski, K. Pakuła, M. Potemski, G. Martinez, I. Grzegory, S. Porowski, Phys. Rev. B 56, 15151 (1997-I).

[5] S.L. Chuang, C.S. Chang, Phys. Rev. B 54, 2491 (1996-II).

[6] W. Shan, B.D. Little, A.J. Fisher, J.J. Song, B. Goldenberg, W.G. Perry, M.D. Bremser, R.F. Davis, Phys. Rev. B 54, 16369 (1996-I).

[7] D.C. Reynolds, D.C. Look, W. Kim, Ö. Aktas, A. Botchkarev, A. Salvador, H. Morkoç, D.N. Talwar, J. Appl. Phys. 80, 594 (1996).

[8] J.F. Muth, J.H. Lee, I.K. Shmagin, R.M. Kolbas, H.C. Casey, Jr., B.P. Keller, U.K. Mishra, S.P. DenBaars, Appl. Phys. Lett. 71, 2572 (1997).

[9] M. Smith, G.D. Chen, J.Y. Lin, H.X. Jiang, M. Asif Khan, C.J. Sun, Q. Chen, J.W. Yang, J. Appl. Phys. 79, 7001 (1996).

[10] M. Steube, K. Reimann, D. Frölich, S.J. Clarke, Appl. Phys. Lett. 71, 948 (1997).

.[11] D. Volm, K. Oettinger, T. Streibl, D. Kovalev, M. Ben-Chorin, J. Diener, B.K. Meyer, J. Majewski, L. Eckey, A. Hoffmann, H. Amano, I. Akasaki, K. Hiramatsu, T. Detchprom, Phys. Rev. B 53, 16543 (1996-II). 